\title{
Zipf's law in Nuclear Multifragmentation and Percolation Theory
}

\author{
Kerstin Paech, Wolfgang Bauer, and Scott Pratt \\ Department of Physics and Astronomy \\ Michigan State University, \\ East Lansing, MI 48824-2320, USA
}

(Dated: October 30, 2018)

\begin{abstract}
We investigate the average sizes of the $n$ largest fragments in nuclear multifragmentation events near the critical point of the nuclear matter phase diagram. We perform analytic calculations employing Poisson statistics as well as Monte Carlo simulations of the percolation type. We find that previous claims of manifestations of Zipf's Law in the rank-ordered fragment size distributions are not born out in our result, neither in finite nor infinite systems. Instead, we find that ZipfMandelbrot distributions are needed to describe the results, and we show how one can derive them in the infinite size limit. However, we agree with previous authors that the investigation of rankordered fragment size distributions is an alternative way to look for the critical point in the nuclear matter diagram.

PACS numbers: 25.70.Pq, 05.70.Jk, 64.60.Ak, 05.70.Fh, 21.65. + f, 25.40.Ve
\end{abstract}

\section{INTRODUCTION}

A central goal of nuclear physics is to study the phase diagram of nuclear and quark-gluon matter. Of special interest are two phase transitions, both of which are expected to be first-order transitions terminating at a critical point in the $\mu-T$ plane. At high temperatures $(T \approx 200 \mathrm{MeV})$ and/or densities QCD tells us that nucleons are no longer bound, but dissolve into assymptotically free quarks and gluons [1], possibly with an accompanying restoration of chiral symmetry [2]. The SPS-CERN and RHIC-BNL experiments have found evidence of this phase transition, and that is might be of first-order (or possibly a cross-over) 3, 4]. Theoretically, lattice-QCD calculations suggest that the nuclear matter phase diagram contains a critical point at high temperature and density $[5,6,6]$. One of the central goals of the future FAIR facility at the German GSI is to study this critical point.

The second transition is under far better control, both experimentally and theoretically, and involves the transition of the low-temperature nuclear Fermi liquid into a gas of nucleons and small fragments. Experimentally $[8,9,10,11,12,13,14]$, one finds a critical point at which this first-order transition terminates. This critical point is located at temperature and density [15, 16, 17]

$$
T_{c}=8.3 \pm 0.2 \mathrm{MeV}, \quad \rho_{c} / \rho_{0}=0.35 \pm 0.1
$$

In addition, through comparisons with phase transition theories, the set of critical exponents determining the universality class of the phase transition has been determined [15]. The experimental values of the exponents, extracted in a somewhat model dependent way, are in good agreement with the universality class of percolation [18, 19, 20, 21, 22, 23], provided that appropriate finite size corrections are included [15, 24, 25]. In passing we note that similar fragmentation patterns pointing to the existence of phase transitions can also be found in the disintegration of molecules, such as buckyballs [26, 27]. With the advent of the very high-powered free-electron lasers at DESY in Hamburg and at SLAC in Stanford a fresh look at these molecular fragmentation experiments will be possible, and first experiments have been proposed [28].

The great majority of analyses of critical phenomena in nuclear fragmentation have considered the shape of the mass distribution, which behaves as a power law in the critical region. In the present article we wish to address whether studying the behavior of the ranked fragment sizes, i.e., the sizes of the largest fragment, second largest, and so on, also contains information regarding critical behavior. As a second goal, we also investigate the applicability Zipf's law, an empirical expression sometimes linked to self-ordered criticality which has recently been applied to nuclear multifragmentation.

\section{ZIPF'S LAW IN NUCLEAR MULTIFRAGMENTION}

When Watanabe [29] and Ma et al. 30, 31] studied the average size of the $n^{\text {th }}$ largest fragment $\left\langle A_{n}\right\rangle$ as a function of $n$ they found that the data is described by Zipf's law 32] which was first found in linguistics and is named after the linguist George Zipf). Zipf's law is empirical, and states that the most frequent word in a given language appears twice as often as the second most frequent word, three times as often as the third most frequent and so on, i.e.

$$
F(r) \propto r^{-1}
$$

where $F(r)$ is the frequency of the $r$-th most frequent word. A more general form,

$$
F(r) \propto r^{-\lambda}
$$

is also often refered to as Zipf's Law. 
Since it was first formulated, many examples outside of linguistics have shown the same behavior. It should also be noted that the Pareto distribution 33] (named after the economist Vilfredo Pareto) contains Zipf's law in a different formulation and was also found in a broad range of statistical data.

Ma et al. claim that since Zipf's law has been observed in very different fields this is a reflection of self-ordered criticality. Therefore, in 30] Zipf's law in its extended form (3) was fitted to experimental data for the average size of the $n^{\text {th }}$ largest fragment, see Figs. 23 and 24 in [30]. They found that for increasing excitation energies $\lambda$ decreases and passes through $\lambda=1$ at some point, i.e. Zipf's law in its original version (2). It is hoped that this passing through $\lambda=1$ is an indication for the critical point.

\section{III. n-TH LARGEST CLUSTERS AND AVERAGE SIZES}

If we assume that the production of any two clusters is largely independent, then the probability that a cluster

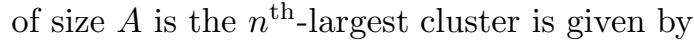

$$
P_{n}(A)=\sum_{i=0}^{n-1} p_{\geq n-i}(A) \cdot p_{i}(>A)
$$

where

$$
p_{\geq m}(A)=1-\sum_{k=0}^{m-1} p_{k}(A)
$$

is the probability to have at least $m$ clusters of size $A$.

Here, $p_{k}(A)$ is the probability to have $k$ clusters of size $A$ and $p_{i}(>A)$ is the probability that there are $i$ clusters of size larger than $A$. For example, the probability that a cluster is the largest in a given fragmentation event is the product of the probability $p_{\geq 1}(A)$ that there is at least one cluster of size $A$ present and the probability $p_{0}(>A)$ that there are zero clusters of size larger than $A$

$$
\begin{aligned}
P_{1}(A) & =p_{\geq 1}(A) \cdot p_{0}(>A) \\
& =\left(1-p_{0}(A)\right) \cdot p_{0}(>A)
\end{aligned}
$$

The probability that a cluster is the second largest is given by a sum of two terms. The first term is the probability $p_{\geq 2}(A)$ that there are two or more clusters of size $A$ present multiplied with the probability $p_{0}(>A)$ that there are no clusters of size larger than $A$. The second term is the probability $p_{\geq 1}(A)$ that there is at least one cluster of size $A$ present multiplied with the probability $p_{1}(>A)$ that there is exactly one cluster of size larger than $A$ present. This yields

$$
\begin{aligned}
P_{2}(A)= & p_{\geq 2}(A) \cdot p_{0}(>A)+p_{\geq 1}(A) \cdot p_{1}(>A) \\
= & \left(1-p_{0}(A)-p_{1}(A)\right) \cdot p_{0}(>A) \\
& +\left(1-p_{0}(A)\right) \cdot p_{1}(>A)
\end{aligned}
$$

for the probability that a cluster is the second largest in a given fragmentation event. From the probability $P_{n^{\text {th }}}$ that a cluster of size $A$ is the $n^{\text {th }}$ largest we can calculate the average size of the $n$-th largest cluster

$$
\left\langle A_{n}\right\rangle=\sum_{A=1}^{V} A \cdot P_{n}(A)
$$

Here, $P_{n}(A)$ depends on the probability distribution $p_{i}$ that is chosen for the underlying physical system. Since we assume that the production of any two clusters is largely independent from one another, the probability distribution can be approximated by the Poisson distribution

$$
p_{k}(A)=\frac{1}{k !}(N(A))^{k} e^{-N(A)},
$$

where $N(A)$ is the appropriate cluster size distribution, i.e. the average of clusters of size $A$, and may depend on additional parameters like the excitation energy of the reaction.

For a system close to the critical point, the cluster size distribution follows a scaling function of the form

$$
n(A, \epsilon)=a A^{-\tau} f\left(\epsilon A^{\sigma}\right),
$$

where $\epsilon$ is the fractional deviation of the control parameter (for example the temperature $T$, i.e. $\left.\epsilon=\left(T-T_{\mathrm{c}}\right) / T_{\mathrm{c}}\right)$ from the critical value. The scaling function $f$ is equal to 1 at the critical point $(f(0)=1)$. The critical exponents $\tau$ and $\sigma$ determine the universality class of the phase transition, and the normalization constant $a$ is defined by the condition that all nucleons belong to some cluster

$$
\sum_{A=1}^{V} A \cdot n(A, \epsilon)=V
$$

where $V$ is the total number of nucleons in an event.

In [34] and 35] the behavior of the average sizes of the $n$-th largest cluster at the critical point $(\epsilon=0)$ was studied assuming that the cluster sizes can be described by a Poissonian probablity distribution. Here, we summarize those results and consider the critical point where the cluster size distribution is a pure power law

$$
N(A)=n(A, 0)=a A^{-\tau},
$$

which with equation (9) can be used to calculate the average size of the $n$-th largest cluster according to equations (44) and (8) and we will consider $2<\tau<3$ in the following. The normalization constant is given by

$$
a=V / \sum_{A=1}^{V} A^{-(1-\tau)}=V / H_{V, 1-\tau}
$$

where $H_{n, m}=\sum_{k=1}^{n} k^{-m}$ is the $n^{\text {th }}$ harmonic number of order $\mathrm{m}$. For the probability to have $i$-clusters larger 
than $A$,

$$
\begin{aligned}
N(>A) & =\sum_{k=A+1}^{V} N(k)=a \sum_{k=A+1}^{V} k^{-\tau} \\
& =a[\zeta(\tau, 1+A)-\zeta(\tau, 1+V)]
\end{aligned}
$$

has to be used with equation (9), where $\zeta(s, q)=\sum_{k=0}^{\infty}(k+q)^{-s}$ is the generalized Riemann function.

For large systems, one can also replace the sum in equations (11) to (15) by an integral. The constant $a$ is then defined by

$$
\int_{A_{\min }}^{V} \mathrm{~d} A a A^{-(\tau-1)}=V
$$

and is given by

$$
a=V(\tau-2)\left(A_{\min }^{-(\tau-2)}-V^{-(\tau-2)}\right)^{-1} .
$$

For the average number of fragments larger then $A$ we obtain

$$
\begin{aligned}
\eta(A) & =\int_{A}^{V} \mathrm{~d} \widetilde{A} a \widetilde{A}^{-\tau} \\
& =\frac{a}{\tau-1}\left(A^{-(\tau-1)}-V^{-(\tau-1)}\right) .
\end{aligned}
$$

The probability that a cluster is the $n^{\text {th }}$ largest is

$$
P_{n}(A)=\frac{\eta^{n-1}}{(n-1) !} e^{-\eta} A^{-\tau}
$$

and therefore the average size of the $n^{\text {th }}$ largest cluster is

$$
\left\langle A_{n}\right\rangle=\int_{A_{\min }}^{V} \mathrm{~d} A A a A^{-\tau} \frac{\eta^{n-1}}{(n-1) !} e^{-\eta} .
$$

We can rewrite this integral by integrating over $\eta(A)$ with

$$
A(\eta)=\left(\frac{\eta(\tau-1)}{a}+V^{-(\tau-1)}\right)^{-\frac{1}{\tau-1}}
$$

and get

$$
\begin{aligned}
\left\langle A_{n}\right\rangle=\left(\frac{a}{\tau-1}\right)^{\frac{1}{\tau-1}} & \int_{0}^{\eta_{\max }} \mathrm{d} \eta\left(\eta+a \frac{V^{-(\tau-1)}}{\tau-1}\right)^{-\frac{1}{\tau-1}} \\
& \times e^{-\eta} \frac{\eta^{n-1}}{(n-1) !}
\end{aligned}
$$

where $\eta(V)=0$ and $\eta_{\max }=\eta\left(A_{\min }\right)$. For average size of the $n+1$-th largest fragment in an infinite system this leads to

$$
\left\langle A_{n}^{\infty}\right\rangle=\left(\frac{a}{\tau-1}\right)^{\frac{1}{\tau-1}} \int_{0}^{\infty} \mathrm{d} \eta e^{-\eta} \frac{\eta^{n-\frac{1}{\tau-1}}-1}{(n-1) !}
$$

which we can express in terms of the gamma function as

$$
\begin{aligned}
\left\langle A_{n}^{\infty}\right\rangle & =\left(\frac{a}{\tau-1}\right)^{\frac{1}{\tau-1}} \frac{\Gamma\left(n-\frac{1}{\tau-1}\right)}{(n-1) !} \\
& =\left(\frac{a}{\tau-1}\right)^{\frac{1}{\tau-1}} \frac{\Gamma\left(n-\frac{1}{\tau-1}\right)}{\Gamma(n)}
\end{aligned}
$$

where in the last step we made use of the fact that $\Gamma(n)=$ $(n-1) !$.

Hence, in the case of an infinite system for $\tau=2$ the average size of the $n^{\text {th }}$ largest cluster is

$$
\left\langle A_{n}^{\infty}\right\rangle=a \frac{\Gamma(n-1)}{\Gamma(n)}=a(n-1)^{-1},
$$

where we used the identity $\Gamma(x+1)=x \Gamma(x)$. Therefore for $\tau=2$ the average size of the $n^{\text {th }}$ largest cluster in an infinite system is not described by Zipf's law, but by the Zipf-Mandelbrot distribution [36, 37]

$$
F(r)=c(r+k)^{-\lambda}
$$

with $k=-1$ and $\lambda=1$. Fig. 1 shows $\left\langle A_{n}\right\rangle$ calculated with equation (8), divided by the normalization constant $a$ for different system sizes $V$. For $n=1$ and $n=2$ the finite size effects are very noticable, however for $n \geq 3$ the $\left\langle A_{n}\right\rangle$ match the Zipf-Mandelbrot distribution. It should also be noted that Zipf's law with $\lambda=1$ does not describe $\left\langle A_{n}\right\rangle$ very well.

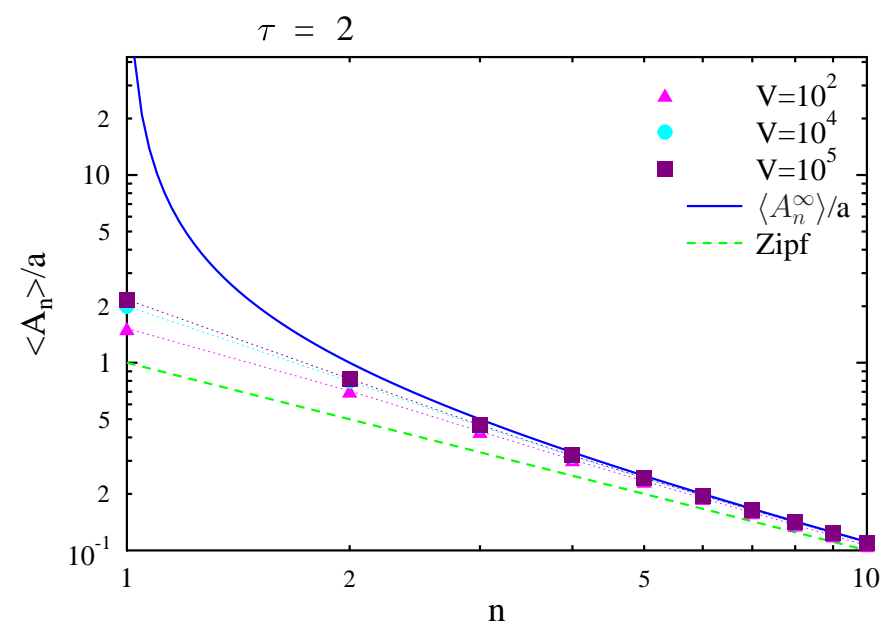

FIG. 1: Symbols indicate $\left\langle A_{n}\right\rangle$ for $\tau=2$ and different system sizes calculated with equation (8), the solid line indicates $\left\langle A_{n}^{\infty}\right\rangle$ from equation (24), the dashed line indicates Zipf's law (2).

Using the ansatz described above we determine $\left\langle A_{n}\right\rangle$ for different values $2 \leq \tau \leq 3$ and test how well they are described by Zipf's law and the Zipf-Mandelbrot distribution.

Fig. 2 shows the extracted fits of the parameter $\lambda$ for Zipf's law for different values $\tau$ and different system sizes 

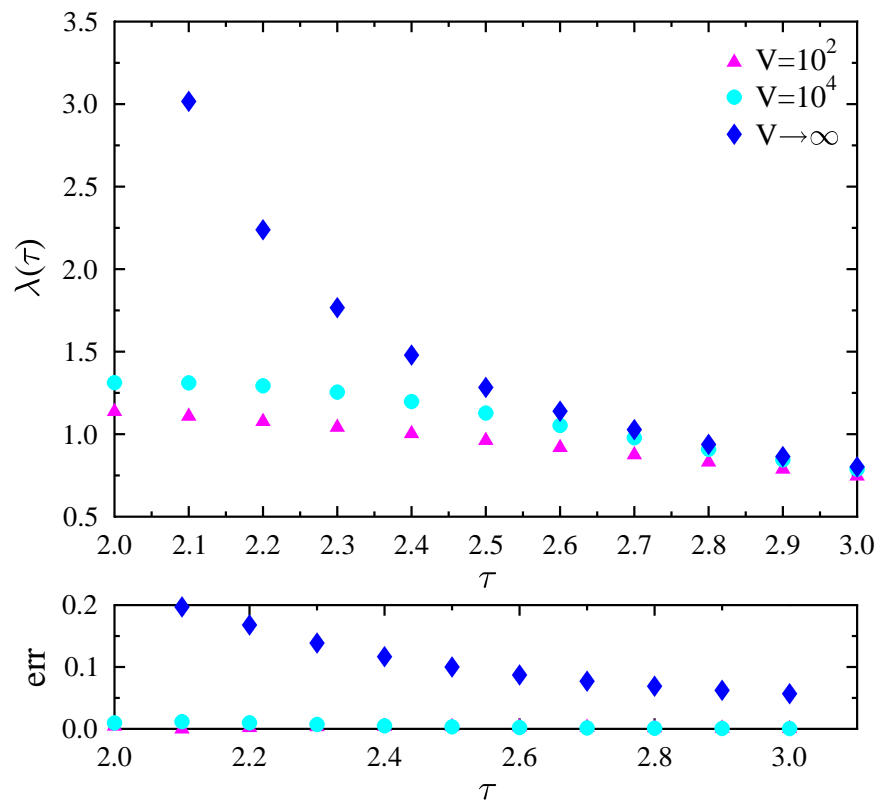

FIG. 2: Top: $\lambda$ as a function of $\tau$ as fitted to Zipf's law (3) for different system sizes. Bottom: Error of the fit

$V$. The value of $\lambda$ for the finite systems shows a decrease from $\lambda(\tau=2)=1.25$ to $\lambda(\tau=3) \approx 0.75$, whereas for the limit of an infinitely large system there is a stronger dependence on $\tau$. The errors of the fit are shown in the lower part of the figure, demonstrating that the fits for the finite systems are a good approximation, while the error for the infinite system is substantially higher. For the finite systems, $\lambda$ is relatively close to unity for the small system with $V=10^{2}$ around $\tau=2.5$. However, for example $\lambda(\tau=3) \approx 0.75$ which would indicate that the statement of 30 that $\lambda=1$ as found in the experimental data is more coincidental than profound. Fig. 2 shows that the value of $\lambda$ at the critical point depends on $\tau$ and the system size $V$ and that $\lambda=1$ at the critical point only holds true for certain sets of parameters.

Fig. 3 shows the extracted fits of the parameter $\lambda$ for the Zipf-Mandelbrot distribution for different $\tau$ and different system sizes $V$. The value of $\lambda$ for the finite systems shows a decrease from $\lambda(\tau=2) \approx 1.2$ to $\lambda(\tau=3) \approx 0.5$, while for the larger system the decrease is not as strong. The difference between the finite systems and the infinite system is not as strong as for the fit to Zipf's law. The errors of the fit are shown in the lower part of the figure showing that the fits for the finite systems are indeed a much better approximation of the $\left\langle A_{n}\right\rangle$ than Zipf's law, and the fit for the infinite system yields the best approximation overall. The parameter $k$ shown in Fig. 3 (middle) shows some finite size dependency for small $\tau$, however the system size dependence decreases with increasing $\tau$.

From fits to the Zipf-Mandelbrot distribution for $2 \leq$ $\tau \leq 3$ one finds a systematic dependence of $\lambda$ on the
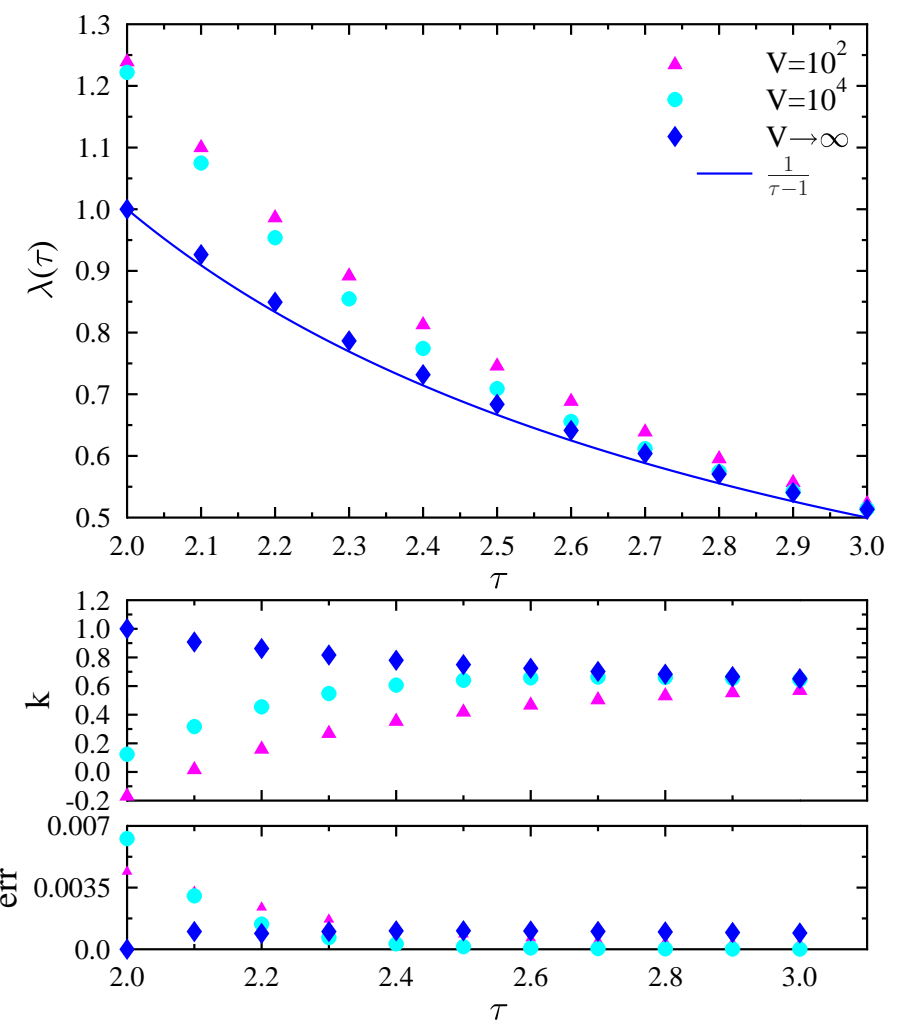

FIG. 3: $\lambda$ as a function of $\tau$ as fitted to the Zipf-Mandelbrot distribution (25) for different system sizes. Middle: Extracted parameter $k$. Bottom: Error of the fit

critical exponent $\tau$ of the form

$$
\lambda \approx \frac{1}{\tau-1},
$$

which holds true almost exaclty for the infinite system and is a good approximation for small systems.

To summarize the findings of this section: Zipf's law should not be applied to nulcear fragmentation, but rather the more general form of the Zipf-Mandelbrot distribution. And $\lambda=1$ as found in the experimental data is more coincidental than a measure of a critical exponent or an indicator of the self-similar behavior.

\section{PERCOLATION THEORY AND ZIPF'S LAW}

The ansatz described in III can only be applied for systems at (or close to) the critical point since information about the scaling function $f$ is needed for $\epsilon \neq 0$. It has been discussed in [18] that bond percolation can be used to describe multi-fragmentation reactions. In the following we will use three dimensional bond percolation model on a simple cubic lattice [38, 39]. The probability to form a bond between two neighboring lattice sites is $p_{\text {bond }}$.

The critical exponents of the percolation model depend on the system size and the dimensionality of the lattice. 
For an infinitely large three dimensional lattice $\tau=2.18$ and $\sigma=0.45$. The critical bond probability does not only depend on the dimensionality of a lattice, but also on its particular topology. For an infinitely large simple cubic lattice the critical probability is $p_{\mathrm{c}}=0.249$.

In the following, we will consider two different system sizes: a small lattice with $6 \times 6 \times 6=216$ sites (corresponding to system sizes found in nuclear fragmentation reactions) and a large lattice with $100 \times 100 \times 100=10^{6}$ sites (reducing the finite size effects considerably).
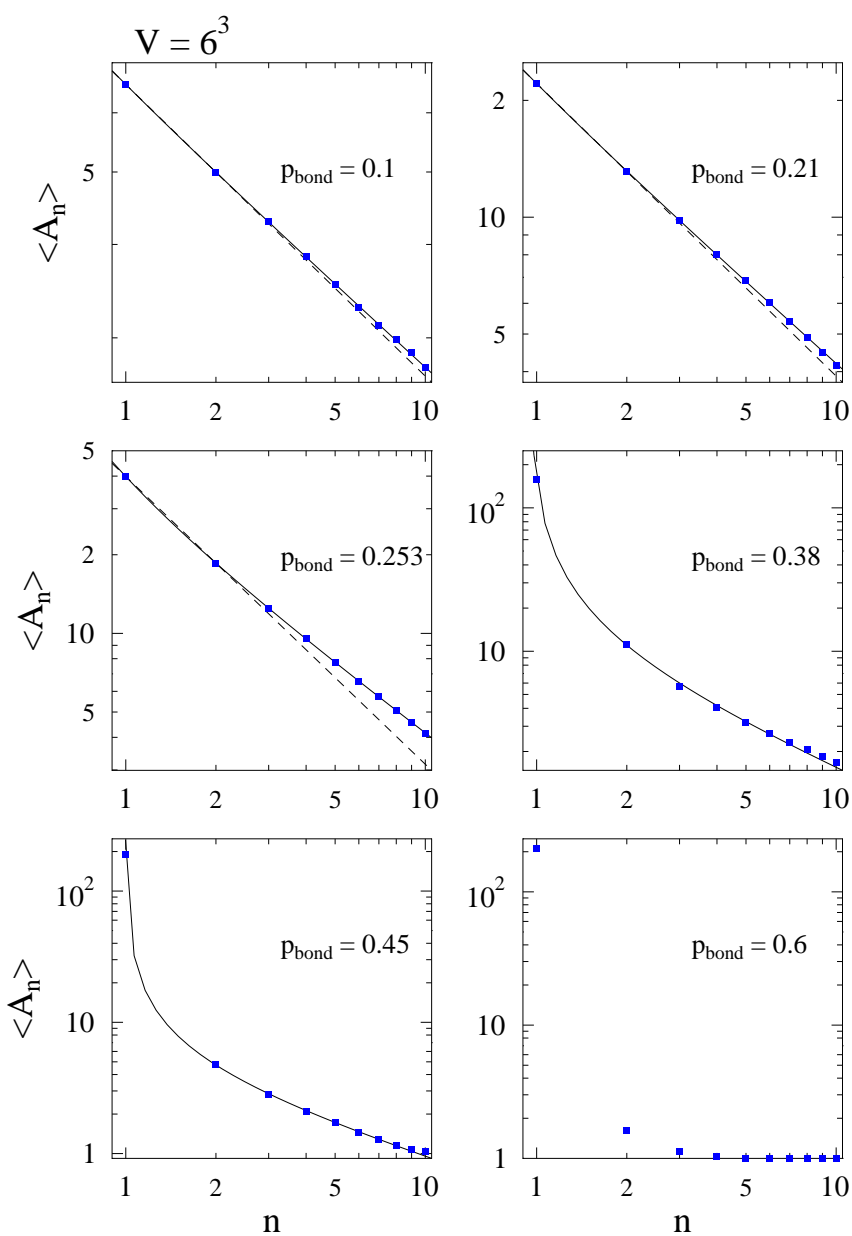

FIG. 4: Average sizes of the $n$-th largest cluster as a function of $n$ for the lattice with 216 sites for different bond probabilities $p_{\text {bond }}$. The dashed lines are fits for Zipf's law (3), the solid lines are fits for the Zipf-Mandelbrot distribution (25).

Fig. 4 shows the average sizes of the $n$-th largest cluster as a function of $n$ for the lattice with 216 sites for different bond probabilities $p_{\text {bond }}$. For this system size we effectively have $\tau \approx 2.14$ at the critical point $p_{\mathrm{c}} \approx 0.253$. The value for $p_{\mathrm{c}}$ was chosen by determining the value of $p_{\text {bond }}$ where the cluster size distributions $N(A)$ were fitted best by the power law distribution (12).

The results for $\left\langle A_{n}\right\rangle$ from percolation theory are similar to the experimental data shown in Fig. 23 from 30]. The small $p_{\text {bond }}$ correspond to high excitation energies and vice versa. For our results from percolation we see that the fits for Zipf's law (and Zipf-Mandelbrot) work very well below $p_{\mathrm{c}}$ (that corresponds to excitation energies above the critical point). But as one crosses the critical point to $p_{\text {bond }}>p_{\mathrm{c}}$ (corresponding to excitation energies above the critical point) we observe that Zipf's law (and eventually Zipf-Mandelbrot) does not work if $\left\langle A_{1}\right\rangle$ is included.

We fit Zipf's law (3) to $\left\langle A_{n}\right\rangle$ from percolation and determined the parameter $\lambda$. Since we use a least squares fitting procedure we use two different methods to obtain a fit. The first method (denoted "Fit 1") is equivalent to what is done for Fig. 24 in [30], i.e. fitting the data to equation (3) with a least square fit. However, this means that for a power law fit the first two data points will dominate the result for the fit. As a second method (denoted "Fit 2") we first take the logarithm of both axis and then fit the $\left\langle A_{n}\right\rangle$ to a straight line to determine $\lambda$, hence all the $\left\langle A_{n}\right\rangle$ contribute more or less equally to the fit result.

We already mentioned that Zipf's law (ZipfMandelbrot) does not work if the average size of the largest fragement $\left\langle A_{1}\right\rangle$ is included above $p_{\mathrm{c}}$. Therefore we perform the two fit methods once including all the fragments and once excluding $\left\langle A_{1}\right\rangle$ from the fit. This way we have four different ways to determine a value for $\lambda$ and the results are shown in Fig. 5 , for both the small and the large lattice.

For the small system we see a similar behavior as for the experimental data from [30] and $\lambda$ ( $\left.p_{\text {bond }}\right) \operatorname{crosses} \lambda=$ 1 at or very close to $p_{\mathrm{c}}$ for both system sizes investigated. The fits are all very similar below $p_{\mathrm{c}}$ as the first fragment hardly deviates from Zipf's law.

In case of the large system $\left(10^{6}\right.$ sites $)$, and for the fit including $\left\langle A_{1}\right\rangle$, a quick rise of $\lambda$ can be observed with increasing $p_{\text {bond }}$ due to the domination of $\left\langle A_{1}\right\rangle$. For the fit excluding $\left\langle A_{1}\right\rangle$ we see a distinct peak of $\lambda\left(p_{\text {bond }}\right)$ close to the critical point.

Although some maximum is visible for the case of the small system (216 sites), the maximum is very broad and not particular near the critical point and can therefore not be taken as an indication of the critical point for these small systems.

If we fit $\left\langle A_{n}\right\rangle$ with the Zipf-Mandelbrot distribution (25), we get a similar behavior for $\lambda$. Further, for the case where we exclude the largest fragment from the fit, the parameter $k$ also indicates the critical point in the case of the large lattice $\left(10^{6}\right.$ sites $)$. Here we observe that $k \approx 0$ for $p_{\text {bond }}<p_{\mathrm{c}}$ and a sharp drop to $k \approx-1$ for $p_{\text {bond }}>p_{\mathrm{c}}$, i.e. the dependence of $k$ can be approximated by

$$
k\left(p_{\text {bond }}\right) \approx-\Theta\left(p_{\text {bond }}-p_{\text {c }}\right) .
$$

This means that we recover Zipf's law if we neglect the largest fragment above $p_{\mathrm{c}}$. However, when turning to the small lattice (216 sites), the sharp drop of $k$ at $p_{\mathrm{c}}$ turns into an extremely smooth decrease over a range in the bond probability of $\Delta p_{\text {bond }} \approx 0.2$. 

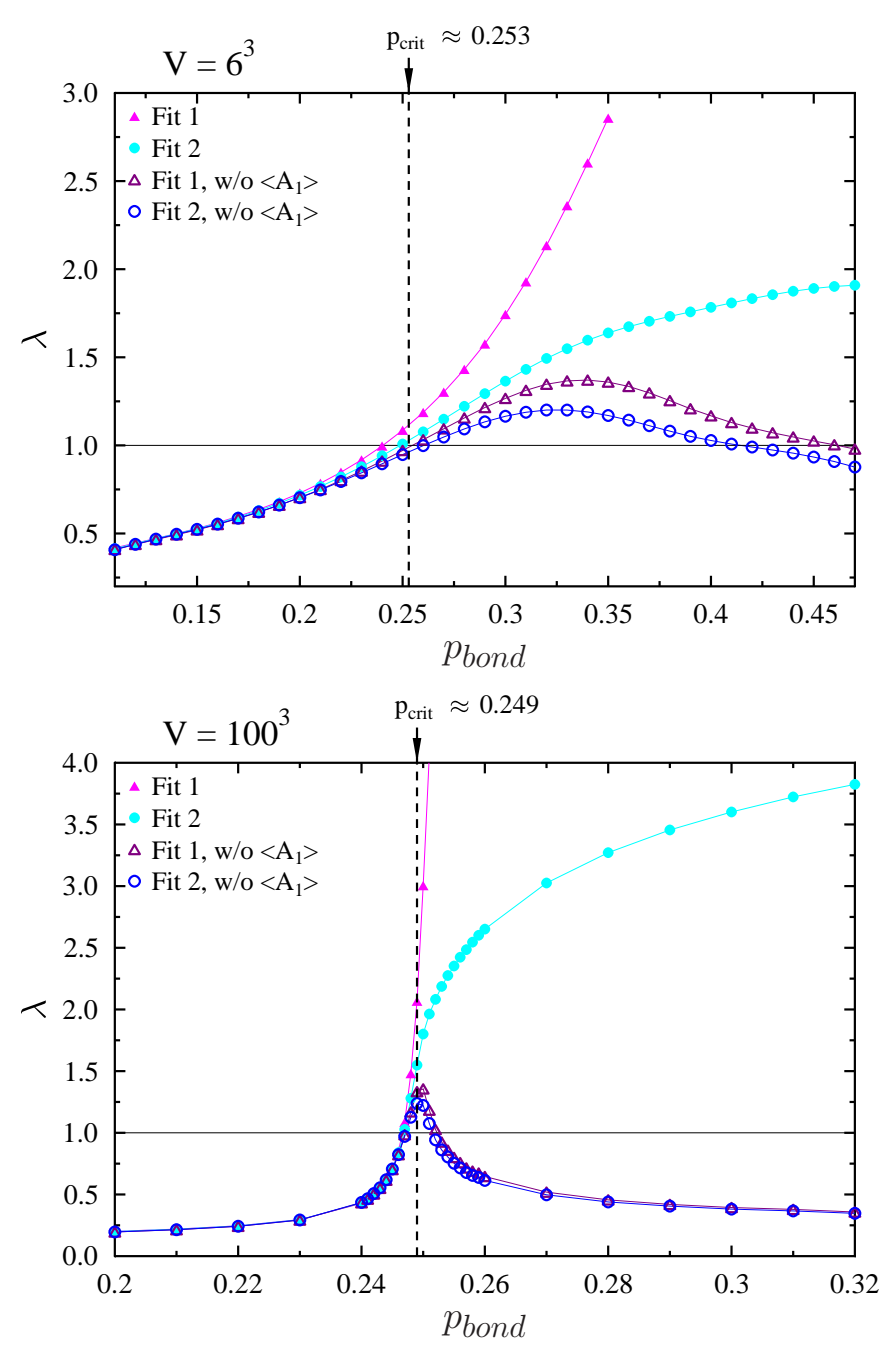

FIG. 5: Fitted values for $\lambda$ using the different fits described in the text. Solid symbols are for fits including the first fragment, open symbols are for fits omitting $\left\langle A_{n}\right\rangle$. Upper figure is for the small lattice (216 sites), lower figure is for the large lattice $\left(10^{6}\right.$ sites $)$. Approximate values for $p_{\mathrm{c}}$ are also indicated by a dashed line for both cases.

Therefore, the crossing through $\lambda=1$ in the experimental data could indeed indicate the crossing through the critical point. However, we should keep in mind the results from sect. III which clarified that the value of $\lambda$ at the critical point depends on $\tau$ and $V$. Therefore, the fact that $\lambda=1$ close to the critical point cannot be taken as a unambigous signature for the critical point.

\section{PERCOLATION THEORY AND $\left\langle A_{n}\right\rangle$}

We have argued in the previous section that the Zipf's law parameter $\lambda$ is not a rigorous signature for the critical point. In this section we want to investigate what can be learned from considering the average size of the $n$-th largest clusters $\left\langle A_{n}\right\rangle$.
We turn to look at the ratio of the $n$-th largest average cluster sizes $\left\langle A_{n+1}\right\rangle /\left\langle A_{n}\right\rangle$. If there is a critical behavior that indicates the critical point we hope to find it by looking at the ratio of the $\left\langle A_{n}\right\rangle$ for different $n$.

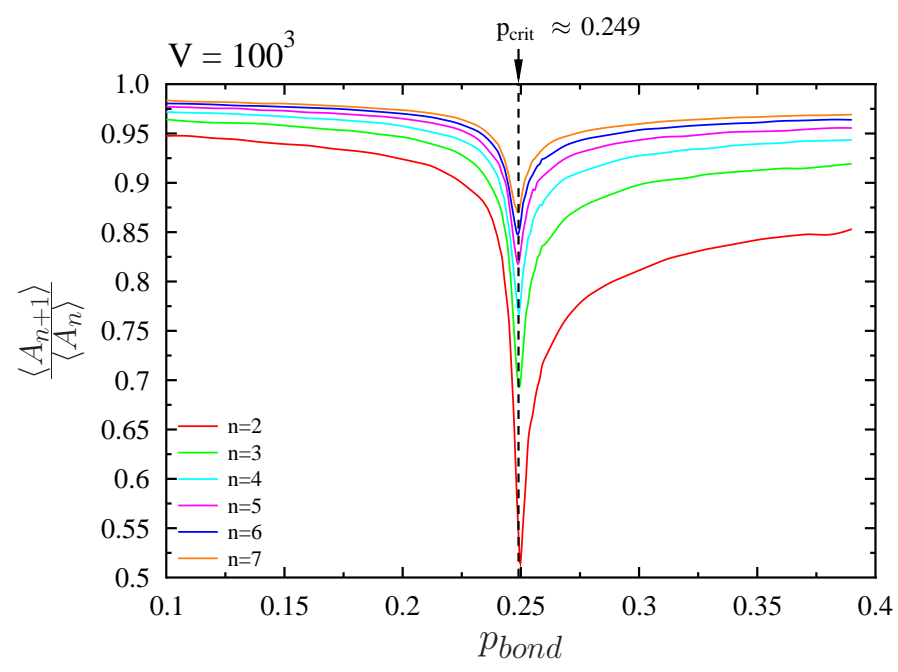

FIG. 6: Ratio of the average size of the $n+1$-th largest fragment to average size of the $n$-th largest fragment as a function of $p_{\text {bond }}$ for a large system with $10^{6}$ sites. The result for $n=1$ is omitted.

Fig. [6] shows the ratio $\left\langle A_{n+1}\right\rangle /\left\langle A_{n}\right\rangle$ (with $n=2 \ldots 7$ ) as a function of $p_{\text {bond }}$ for the large system $\left(10^{6}\right.$ sites $)$. Close to the critical point all ratios show a significant minimum at the critical bond probability $p_{\mathrm{c}}$. The average size of the largest fragment $\left\langle A_{1}\right\rangle$ has a very different behavior compared to $\left\langle A_{n}\right\rangle$ for $n>1$ and is therefore omitted.

Now we want to compare the results shown in Fig. 6 to the ansatz from section [II] for an infinite system. In section [II two assumptions were made: at the critical point the cluster size distributions are discribed by a power law and the production of the clusters is uncorrelated and therefore Poissonian.

For percolation theory we also expect to have a power law for the cluster size distributions at the critical point. Therefore, the only difference we would expect at the critical point compared to the ansatz from section III for an infinite system besides finite size effects, would be due to correlations. These correletions would stem from the fact that the production of large clusters is not independent. For example, if a single very large cluster is produced whose size is of the order of the total sytem size no further large clusters can be produced in the same event. However, the production of the smaller clusters would not be affected. Hence, at the critical point we wouldn't expect the Poissonian ansatz to work for the ratio $\left\langle A_{n+1}\right\rangle /\left\langle A_{n}\right\rangle$ for $n=1$, but possibly for $n$ larger than 2 or 3 .

Before comparing the percolation results to the ansatz from section III for an infinite system we first want to make sure that finite size effects are negligible. Fig. 7 
shows the ratio $\left\langle A_{n+1}\right\rangle /\left\langle A_{n}\right\rangle$ for different system sizes. It

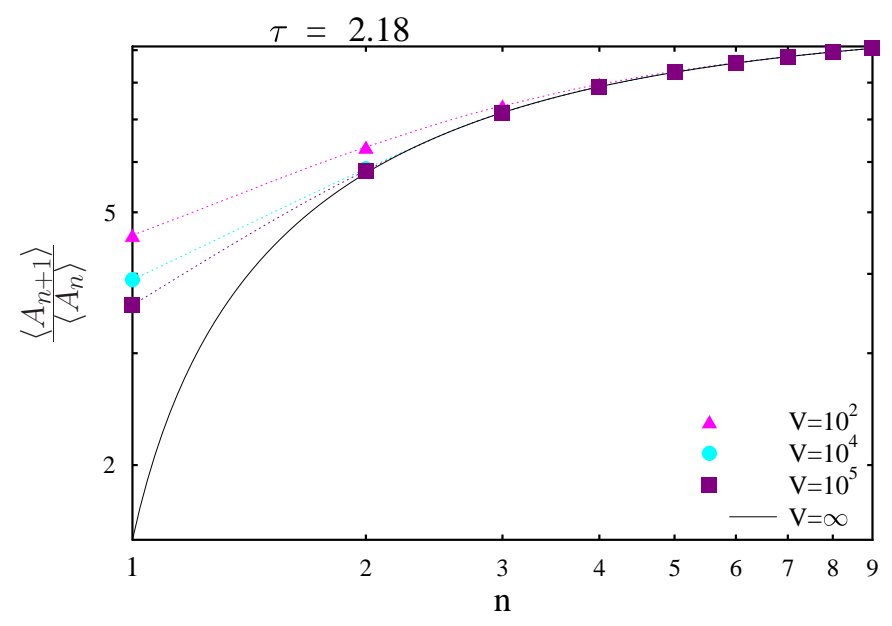

FIG. 7: The ratio $\left\langle A_{n+1}\right\rangle /\left\langle A_{n}\right\rangle$ for different finite system sizes $V$ (symbols) using eqn. (8) and for the infinte system (line) using eqn. (23) for $\tau=2.18$.

shows that finite size effects between the infinite system and the finite system sizes are negligible for $n>1$ for the system sizes larger than $V=10^{2}$, and for $n>2$ are negligible for all shown system sizes. Therefore, the ansatz from section III for an infinite system can be used for the comparison to the percolation model if the ratio for $n=1$ (and $n=2$ for the smaller system) is excluded. However, it should be noted that for $\tau=2$ the finite size effects are again substantial for $n=1$ and non-negligible for $n=2$ and $n=3$, the finite size effects get negligible for $n>3$, as is shown in Fig. 8

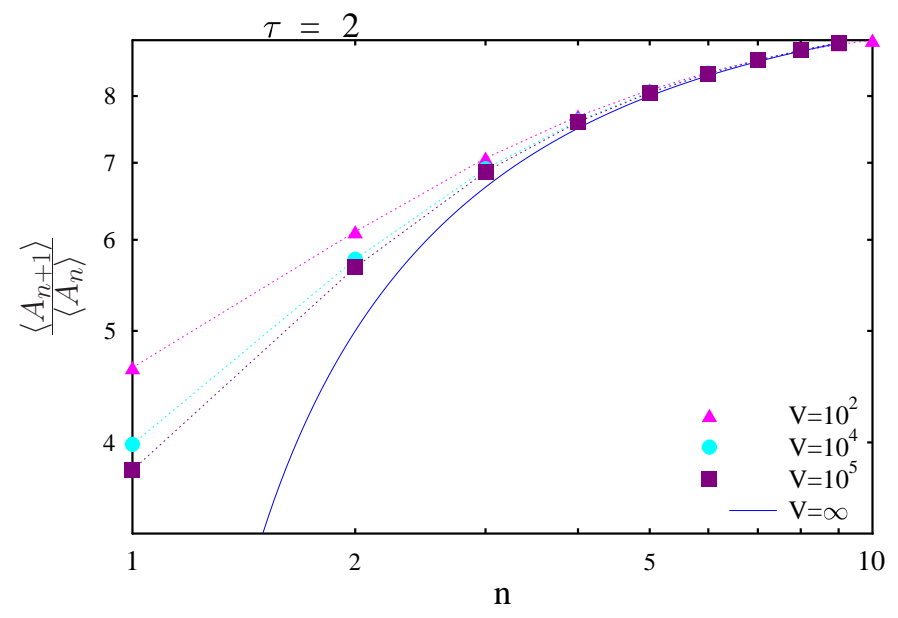

FIG. 8: The ratio $\left\langle A_{n+1}\right\rangle /\left\langle A_{n}\right\rangle$ for different finite system sizes $V$ (symbols) using eqn. (8) and for the infinte system (line) using eqn. (24) for $\tau=2$.

To compare the percolation result to the results from ansatz from section [II that resulted in equation (23). Using the identity $\Gamma(x+1)=x \Gamma(x)$ we can write the ratio as

$$
\frac{\left\langle A_{n+1}^{\infty}\right\rangle}{\left\langle A_{n}^{\infty}\right\rangle}=\frac{n(\tau-1)-1}{n(\tau-1)}
$$

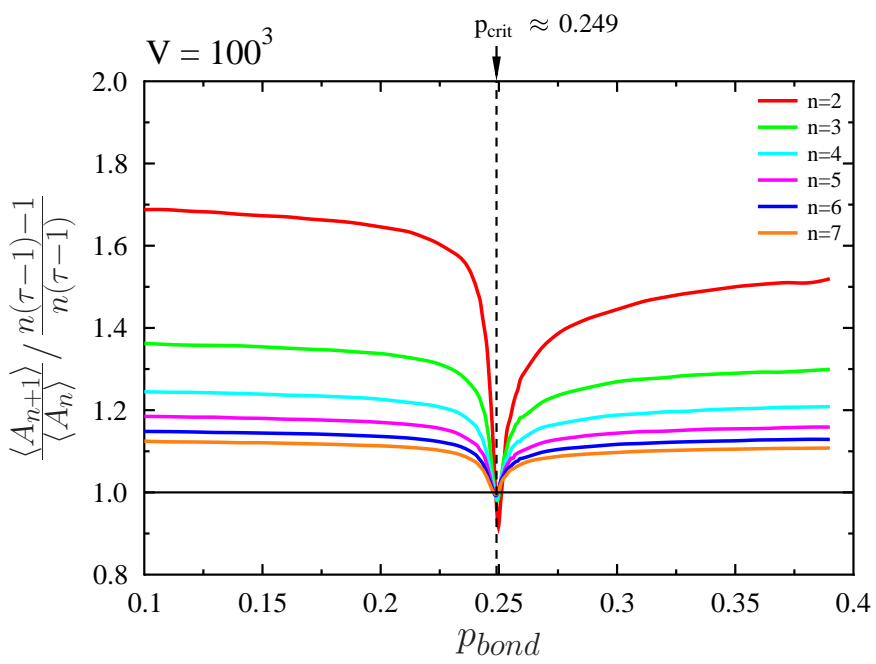

FIG. 9: Ratio of the average size of the $n+1$-th largest fragment to average size of the $n$-th largest fragment as a function of $p_{\text {bond }}$ normalized by the factor in equation (28) for a large system $\left(10^{6}\right.$ sites $)$. The result for $n=1$ is omitted.

The ratio of the percolation result and the ansatz from section [II as a function of $p_{\text {bond }}$ is shown in Fig. 9] The minima all come down to 1 for $n \geq 3$ and one can conclude that for the percolation model (at the critical point where the fragments are distributed according to a power law) the production of the $n$-th largest cluster with $n \geq 2$ is not correlated for a large system.

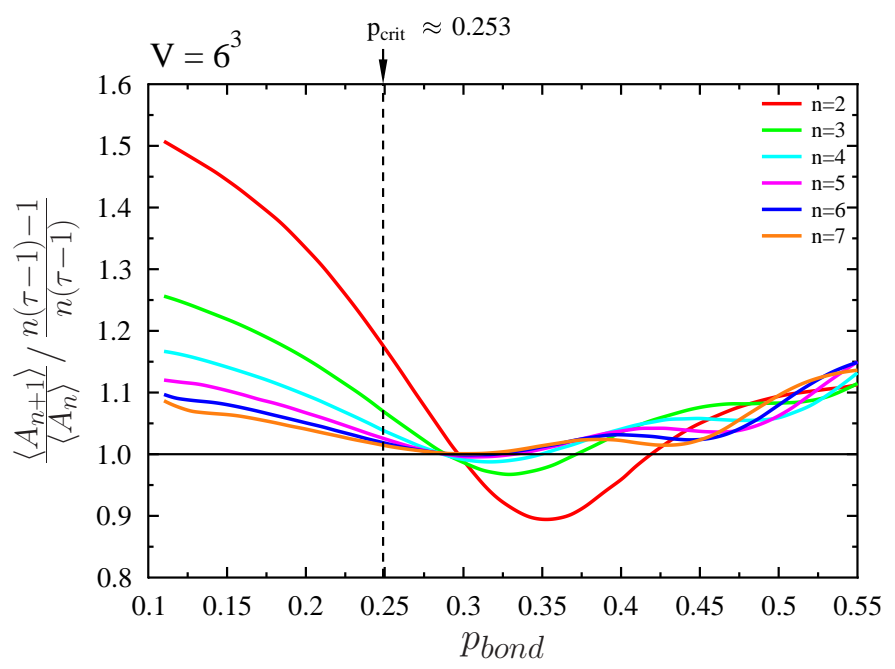

FIG. 10: Ratio of the average size of the $n+1$-th largest fragment to average size of the $n$-th largest fragment as a function of $p_{\text {bond }}$ normalized by the factor in equation (28) for a small system (216 sites). The result for $n=1$ is omitted. 
When turning to the small system (216 sites) shown in Fig. 10 however, the strong indication of the critical point by the location of the pronounced minima does not hold true anymore. We observe a broadening of the minima and a shift of the minima away from the critical point towards higher $p_{\text {bond }}$. For $n \geq 5$ the minima still meet at some $p_{\text {bond }}$, but it is not located close to $p_{\mathrm{c}} \approx 0.253$. This means that the percolation model and ansatz from section 【II agree for values of $p_{\text {bond }}$ where the cluster distribution is no longer a pure power law.

\section{SUMMARY}

In contrast to previous claims, we have shown that a strict application of Zipf's law to rank-ordered fragment size distributions near the critical point of the nuclear fragmentation phase transition is not warranted. This was done in analytical calculations assuming independent Poisson emission probabilities as well as in calculations based on percolation models, which include all correlations. In order to obtain information on finite-size effects, we have performed these calculations over a wide variety of system sizes, and compared to analytical results for infinite systems. We have found that the rank-ordered fragment size distributions follow the more general ZipfMandelbrot distributions instead of the simple Zipf law, and we have been able to derive this relation in the infinite size limit for $\tau$ approaching 2 .

For very large system sizes, we also find that monitoring the value of the Zipf-Mandelbrot distribution fit to the rank-ordered fragment sizes yields a valuable signal for the detection of the critical point in the phase diagram. However, for the extremely small systems typical of nuclear fragmentation we find that this signal is not nearly as reliable as the previously explored scaling analysis [15, 40, 41].

\section{Acknowledgments}

This research was supported by NFS grant PHY0555893 (WB) and the U.S. Department of Energy, Grant No. DE-FG02-03ER41259 (SP). KP greatfully acknowledges support by the Feodor Lynen Program of the Alexander von Humboldt Foundation.
[1] J. C. Collins and M. J. Perry, Phys. Rev. Lett. 34, 1353 (1975).

[2] F. Wilczek, Phys. Today 53N8, 22 (2000).

[3] J. W. Harris and B. Muller, Ann. Rev. Nucl. Part. Sci. 46, 71 (1996).

[4] B. Muller and J. L. Nagle, Ann. Rev. Nucl. Part. Sci. 56, $93(2006)$.

[5] F. Weber, J. Phys. G27, 465 (2001).

[6] Z. Fodor and S. D. Katz, JHEP 03, 014 (2002).

[7] Z. Fodor and S. D. Katz, JHEP 04, 050 (2004).

[8] A. S. Hirsch et al., Phys. Rev. C29, 508 (1984).

[9] M. L. Gilkes et al., Phys. Rev. Lett. 73, 1590 (1994).

[10] H. G. Ritter et al., Nucl. Phys. A583, 491C (1995).

[11] J. B. Elliott et al., Phys. Rev. Lett. 85, 1194 (2000).

[12] T. Lefort et al., Phys. Rev. Lett. 83, 4033 (1999).

[13] L. Beaulieu et al., Phys. Lett. B463, 159 (1999).

[14] L. Beaulieu et al., Phys. Rev. C63, 031302 (2001).

[15] M. Kleine Berkenbusch et al., Phys. Rev. Lett. 88, 022701 (2002).

[16] W. Bauer, B. Alleman, and S. Pratt, Proceedings for IWM 2005, Catania, Italy, 279 (2005).

[17] W. Bauer, Nuclear Physics A 787, 595c (2007).

[18] W. Bauer, U. Post, D. R. Dean, and U. Mosel, Nucl. Phys. A452, 699 (1986).

[19] W. Bauer, D. Dean, U. Mosel, and U. Post, Phys. Lett. B150, 53 (1985).

[20] X. Campi, Journal of Physics A: Mathematical and General 19, L917 (1986).

[21] T. S. Biro, J. Knoll, and J. Richert, Nuclear Physics A 459, 692 (1986).

[22] J. Nemeth, M. Barranco, J. Desbois, and C. Ngo, Z. Phys. A325, 347 (1986).

[23] W. Bauer, Phys. Rev. C 38, 1297 (1988).
[24] W. Bauer and A. Botvina, Phys. Rev. C55, 546 (1997).

[25] W. Bauer and A. Botvina, Physical Review C 52, R1760 (1995)

[26] T. LeBrun, H. G. Berry, S. Cheng, R. W. Dunford, H. Esbensen, D. S. Gemmell, E. P. Kanter, and W. Bauer, Phys. Rev. Lett. 72, 3965 (1994).

[27] S. Cheng, H. G. Berry, R. W. Dunford, H. Esbensen, D. S. Gemmell, E. P. Kanter, T. LeBrun, and W. Bauer, Phys. Rev. A 54, 3182 (1996).

[28] N. Berrah, private communication.

[29] M. S. Watanabe, Phys. Rev. E 53, 4187 (1996).

[30] Y. G. Ma et al., Phys. Rev. C71, 054606 (2005).

[31] Y.-G. Ma, Phys. Rev. Lett. 83, 3617 (1999).

[32] G. K. Zipf, Human Behavior and the Principle of Least Effort (Addison-Wesley, Cambridge, MA, 1949).

[33] V. Pareto, Cours d'économie politique (Droz, Genève, 1897), Vol. 2, reprinted as a volume of Oeuvres Complètes.

[34] W. Bauer, S. Pratt, and B. Alleman, AIP Conf. Proc. 884, 327 (2007).

[35] X. Campi and H. Krivine, Phys. Rev. C72, 057602 (2005).

[36] B. B. Mandelbrot, The Fractal Geometry of Nature (W. H. Freeman, New York, 1982).

[37] M. Mandelbrot, in Communication Theory, edited by W. Jackson (Acad. Press N.Y., London, 1953), pp. 486-502.

[38] G. Grimmett, Percolation, 1989.

[39] D. Stauffer and A. Aharony, Introduction to Percolation Theory: Second Edition (Taylor and Francis, London, Washington DC, 1992).

[40] J. B. Elliott et al., Phys. Rev. Lett. 88, 042701 (2002).

[41] C. M. Mader et al., Phys. Rev. C68, 064601 (2003). 